\title{
Paper Tractography Methods in Preoperative Neurosurgical Planning
}

\author{
Mateusz Koryciński and Konrad A. Ciecierski \\ Department of Bioinformatics and Machine Recognition, \\ Research and Academic Computer Network, Warsaw, Poland
}

https://doi.org/10.26636/jtit.2021.154521

\begin{abstract}
Knowledge of the location of nerve tracts during the surgical preoperative planning stage and during the surgery itself may help neurosurgeons limit the risk of causing neurological deficits affecting the patient's essential abilities. Development of MRI techniques has helped profoundly with in vivo visualization of the brain's anatomy, enabling to obtain images within minutes. Different methodologies are relied upon to identify anatomical or functional details and to determine the movement of water molecules, thus allowing to track nerve fibers. However, precise determination of their location continues to be a labor-intensive task that requires the participation of highly-trained medical experts. With the development of computational methods, machine learning and artificial intelligence, many approaches have been proposed to automate and streamline that process, consequently facilitating image-based diagnostics.

This paper reviews these methods focusing on their potential use in neurosurgery for better planning and intraoperative navigation.
\end{abstract}

Keywords-artificial intelligence, diffusion tensor imaging, Dijkstra's algorithm, graph traversing, MRI, neural networks, tractography.

\section{Introduction}

The human body is controlled by the central nervous system, with the brain being a crucial organ of that system. Brain cortex is made of neural cell bodies tasked with processing information and performing cognitive process. The axons of these cells constitute the white matter that is located underneath and connects various areas of the cortex and transmit nerve impulses between them. All those structures are organized into complex neural circuits, serving as a platform for all vital body functions, such as cognitive processes, movement, sight, as well as production and comprehension of speech [1].

Like the rest of the body, the central nervous system is susceptible to numerous illnesses, including Alzheimer's disease, Parkinson's disease or brain cancers, such as glioma. Neurosurgery is the primary treatment in dealing with gliomas [2]. Although neurosurgery is invasive and risky, in many cases it is the only way to extend the patient's life and to improve the quality of their life. Prior to the surgery, the patient undergoes a series of tests, including magnetic resonance imaging (MRI), with a view of mitigating potential risks. Data collected through this method is used to annotate functional areas of the cortex and to determine the location of nerve pathways connecting them. Although the anatomy of the brain is fairly well known, the precise location of functional areas varies from patient to patient. Furthermore, it has been shown that functional areas may change their location over time. Damage caused to any of the functional areas or nerve pathways may lead to complications and irreversible neurological deficits, making imaging necessary before each surgery (Fig. 1).

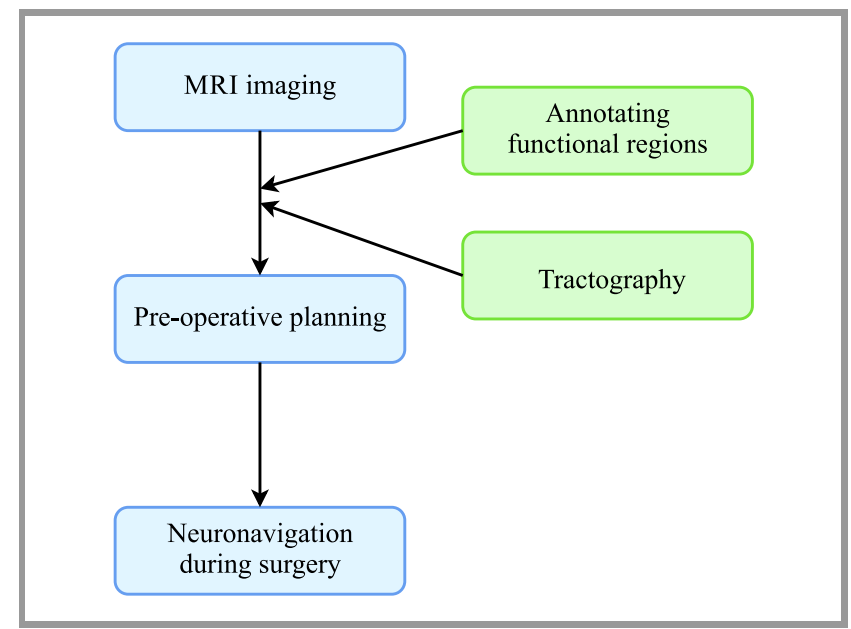

Fig. 1. Outline of the procedure including imaging studies and planning before neurosurgical intervention.

Surgeons may be guided by such knowledge in preoperative planning, deciding on the scope of the intervention and the appropriate entry point. In addition, doctors may use such data during the operation itself, using it as a source of precise information about the layout of the operating field. Tractography is a technique that emerged at the beginning of this century to determine the topology of white matter fibers (nerve tracts) within the brain. Diffusionweighted magnetic resonance imaging (DW-MRI) [3], [4] uses a specific sequence of pulses and field gradients to produce images where diffusion of water molecules generates the contents. Consequently, it allows to observe, 
non-invasively, the process of water diffusion in the living cells. The process of diffusion is anisotropic due to the presence of numerous obstacles in the cells, including organelles and cell membranes [5], [6]. In the axons, it is the myelin sheath that serves as the primary barrier, causing water to travel along the fibers. Hence, tracking water diffusion in the white matter reveals its delicate structure within the brain [7]. The Human Connectome Project [8], a publicly funded multi-institutional initiative, has undertaken the task of constructing a comprehensive map of structural and functional neural connections in vivo. An atlas of human brain connectivity is one of the outcomes of the project [9].

Many algorithms have been proposed to produce tractograms [10], [11]. The difficulty in developing the ideal solution lies in numerous problems encountered when analyzing the data, i.e. fiber crossings, false continuities, fiber truncation, as well as when choosing relevant stopping rules or accounting for the presence of edema noise.

This paper provides a review of specific methods that may potentially be used for fiber annotation in neurosurgical applications. We survey primarily algorithms that are suitable for local tractography, as such an approach restricts our analysis to a particular area. In such applications, both deterministic and probabilistic methods may be used. The source of the data is a very important consideration, as it may be a limiting factor affecting clinical applications. Although diffusion tensor imaging (DTI) [12] sequences are usually relied upon, we decided to highlight the advantage of using high-angular resolution DW imaging (HARDI) [13], [14] by focusing on algorithms using this type of data. In the following sections, we describe classical and artificial intelligence methods, then proceeding to the presentation of benchmarking studies. Then, we describe our case study that focuses on tractography in preoperative neurosurgical planning.

\section{Mathematical Models}

Mathematical models are deemed to be the results of methods used for predicting the orientation of fibers without any support from machine learning algorithms, including from neural networks. Such methods make assumptions based solely on mathematical concepts helping predict local or global tractograms based on diffusion-related data.

Deterministic algorithms focus on envisioning the orientation of a nerve fiber through a single voxel consideration, by expanding the tract from a defined seed point [11]. Seed points are drawn directly from a region of interest (ROI). In neurosurgery, an ROI may be defined as the area of the planned intervention, especially if any part thereof it is located on the border between gray and white matter, healthy and tumorous tissue. It may also be defined as a relevant functional region identified with the help of functional magnetic resonance imaging.

Just like their deterministic counterparts, probabilistic models may start from a given seed point. However, they do not follow a single tract. Instead, they keep on building a distribution of probable streams. Such algorithms are computationally more expensive than those relied upon in the deterministic approach. However, they are better suited for tracking in high uncertainty regions (e.g. crossing fibers), especially when noise is present, which is where deterministic approaches are prone to fail [11]. All methods described below are compared in Table 1 in which such factors as diffusion data source, approach to tracking, key underlying concepts and stopping criteria are taken into consideration.

\subsection{Linear Forced Vector Differential Equation}

One of the first attempts was made in the previous century by Basser in [15]. His methodology generates a dif-

Table 1

Comparison of different classical models for solving tractography tasks

\begin{tabular}{|l|l|l|l|l|}
\hline Method & Data source & Approach & Key concepts & Stopping criteria \\
\hline Basser & DTI & Deterministic & $\begin{array}{l}\text { Solving linear forced vector } \\
\text { differential equation with } \\
\text { Taylor series approximation }\end{array}$ & Not discussed by the author \\
\hline TEND & DTI & Deterministic & $\begin{array}{l}\text { Incoming vector deflection } \\
\text { determining local orientation }\end{array}$ & $\begin{array}{l}\text { Fractional anisotropy below } \\
\text { certain threshold; change in the } \\
\text { direction exceeding 45 }\end{array}$ \\
\hline MRtrix & HARDI & $\begin{array}{l}\text { Deterministic, } \\
\text { probabilistic }\end{array}$ & $\begin{array}{l}\text { Tracking based on fiber } \\
\text { orientation function }\end{array}$ & $\begin{array}{l}\text { FOD peaks below certain } \\
\text { threshold; fiber located outside } \\
\text { ROI }\end{array}$ \\
\hline $\begin{array}{l}\text { Complex fiber } \\
\text { orientation } \\
\text { distribution }\end{array}$ & HARDI & $\begin{array}{l}\text { Deterministic, } \\
\text { probabilistic }\end{array}$ & $\begin{array}{l}\text { Utilizes ODF form Q-ball } \\
\text { imaging and ODF from } \\
\text { a sharpening deconvolution } \\
\text { transform }\end{array}$ & $\begin{array}{l}\text { Tracked fiber located outside } \\
\text { white matter area }\end{array}$ \\
\hline $\begin{array}{l}\text { Hough transform } \\
\text { voting }\end{array}$ & $\begin{array}{l}\text { DSI, DTI, } \\
\text { HARDI }\end{array}$ & Probabilistic & $\begin{array}{l}\text { Selecting the most probable } \\
\text { fibers based on Hugh transform } \\
\text { voting procedure }\end{array}$ & $\begin{array}{l}\text { Tracking is not performed } \\
\text { outside specified user-defined } \\
\text { area }\end{array}$ \\
\hline
\end{tabular}


fusion tensor field from DTI used to obtain fiber trajectory at a given point by solving a linear forced vector differential equation. Nearby points are approximated using the Taylor series expansion. The method uses the eigenvector direction for the highest eigenvalue, assuming that it precisely describes precisely the direction of water diffusion at a given point.

\subsection{Tensor Deflection}

The TEND algorithm developed by Lazar et al. [16] uses the tensor deflection technique to estimate fiber trajectory. In contrast with the method by Basser et al. [15], the whole diffusion tensor is used, not only the eigenvector with the highest eigenvalue. Tract reconstruction is performed in a stepwise fashion, where tract direction from the previous step is treated as an incoming vector. This vector is then deflected by the tensor operator towards the major eigenvector direction.

The resulting vector describes the orientation of the fiber at a given position. The curvature of the deflection is limited, resulting in much smoother tract reconstruction. Tracking with TEND is terminated when fractional anisotropy drops below a certain threshold value, or when the change in the direction exceeds $45^{\circ}$.

\subsection{MRtrix}

MRtrix [17] is a freely available software that combines multiple tools for performing tractography-related tasks. It requires data collected from HARDI imaging. Such data allows to determine fiber orientation by applying constrained spherical deconvolution (CSD) [18] in each voxel to produce a fiber orientation density function (FOD). The resulting FOD holds all information about orientations within a single voxel and is ideal for tracking algorithms. MRtrix relies on two of them a deterministic and a probabilistic one. The deterministic algorithm reconstructs a single fiber along its local orientation. Authors use the NewtonRaphson gradient ascent algorithm to identify the nearest peak in the FOD data iteratively. The peak identification procedure is run once per point, resulting in the most closely aligned direction of the peak, as the current orientation indicates.

In the probabilistic variant, the direction of the next step is provided by a random sampling of the FOD. Sampling is restricted to directions within a certain angle from the current orientation. The selected sample is used to guide towards the next step, as long as the amplitude exceeds a certain threshold. Otherwise, a new sample is generated, and the process is repeated the number of times specified by the user. In comparison to other methods, smoothness and good resolution are achieved by using different data sources and a step size that is smaller than the voxel size. Both algorithms stop tracking when either of the two criteria is met - no satisfying FOD peak can be found (with an amplitude above a certain, user-defined threshold) or when tract propagation reaches the area outside the specified ROI.

\subsection{Complex Fiber Orientation Distribution}

The method developed by Descoteaux et al. [19] uses data from HARDI experiments as well. Their approach uses the sharpening deconvolution function (SDT) to the orientation distribution function (ODF) from Q-ball imaging [20]. The sharpening operation is required due to sparsity of the fiber ODF and the fact that diffusion represented by Q-ball differs from the real direction of the nerve fiber. As it is the case with the MRtrix package, authors propose both deterministic and probabilistic tracking algorithms. The deterministic approach extends classical streamline techniques by considering multiple ODF maxima at each step, where the direction is chosen from 1281 possibilities. The winning direction exceeds all its neighbors' peak values and features an ODF value above 0.5.

The probabilistic algorithm is an interesting extension of the random walk method [21], [22], exploiting the information in multidirectional fiber ODF. Fiber search is performed by particles moving freely from a given seed point, according to the local ODF information. Each voxel is scored based on the number of particles that have reached it. Fiber direction is chosen out of 120 discrete directions, based on the probability derived from the voxel scores, with the step size of 0.5 the voxel size. The procedure is terminated once the particle leaves the white matter. Anisotropy measure map allows the detection of such an event, which is a very precise stopping criterion.

\subsection{Hough Transform Voting}

Aganji et al. [23] propose to use a voting process based on Hugh transform to determine the global topology of white matter tracts within the brain or within a selected ROI. Even though global tractography is not optimal in neurosurgical applications, we decided this method was worth mentioning for two reasons. Firstly, even when we consider that the main aim is to obtain the global tractogram, the user can still impose constraints on the area where tracking occurs. Secondly, the method is developed to work with data from various DWI modalities, such as diffusion spectrum imaging (DSI) [24], DTI or HARDI. In the search space mentioned above, i.e. the whole brain in non-restricted tractography, random seed points are drawn. Curves passing through these points are parametrized by the length of the arc, representing potential fiber orientations. Each one is then scored based on the probability of each curve being part of the same fiber as the seed point. Finally, those with the highest scores are selected as the most probable anatomic connections.

The described approach is an exhaustive search, capable of avoiding local minima. There is no clear stopping criterion except of the ROI area constraint and the brain surface in the global variant.

\section{Artificial Intelligence}

Machine learning (ML) techniques have been successfully applied in many areas of our daily lives, such as spam 
Table 2

Comparison of different machine learning approaches used to solve tractography-related tasks

\begin{tabular}{|c|c|c|c|c|}
\hline Method & Algorithm & Approach & Key concepts & Stopping criteria \\
\hline $\begin{array}{l}\text { Random forest } \\
\text { classification } \\
\text { using neighborhood } \\
\text { information } \\
\end{array}$ & $\begin{array}{l}\text { Random forest } \\
\text { classifier }\end{array}$ & Probabilistic & $\begin{array}{l}\text { Voting process deciding } \\
\text { between probable directions } \\
\text { or termination }\end{array}$ & $\begin{array}{l}\text { Tracking stops when nonfiber } \\
\text { probability exceeds cumulated } \\
\text { weighted probabilities for } \\
\text { continuation directions }\end{array}$ \\
\hline Learn-to-track (1) & $\begin{array}{l}\text { Feed-forward } \\
\text { neural network }\end{array}$ & Deterministic & $\begin{array}{l}\text { Outputs three-dimensional } \\
\text { normalized vector, describing } \\
\text { the streamline direction }\end{array}$ & Not implemented \\
\hline Learn-to-track (2) & $\begin{array}{l}\text { Recurrent } \\
\text { neural network }\end{array}$ & Deterministic & $\begin{array}{l}\text { Neural network architecture } \\
\text { allows to consider previously } \\
\text { seen points to better predict } \\
\text { the fiber topology }\end{array}$ & Not implemented \\
\hline $\begin{array}{l}\text { Bundle-wise deep } \\
\text { tracker }\end{array}$ & $\begin{array}{l}\text { Recurrent } \\
\text { neural network }\end{array}$ & Deterministic & $\begin{array}{l}\text { Trained to predict tracking } \\
\text { direction from a bundle-wise- } \\
\text { input data }\end{array}$ & No information \\
\hline DeepTract & $\begin{array}{l}\text { Recurrent } \\
\text { neural network }\end{array}$ & $\begin{array}{l}\text { Deterministic, } \\
\text { probabilistic }\end{array}$ & $\begin{array}{l}\text { Estimating local fiber orien- } \\
\text { tation as a discrete probability } \\
\text { density function }\end{array}$ & $\begin{array}{l}\text { Track termination probability } \\
\text { as one of the predict classes }\end{array}$ \\
\hline $\begin{array}{l}\text { Neural network } \\
\text { regression }\end{array}$ & $\begin{array}{l}\text { Multilayer } \\
\text { perceptron }\end{array}$ & Deterministic & $\begin{array}{l}\text { Predicting fiber orientation } \\
\text { in a cube of a given size based } \\
\text { on diffusion data and existing } \\
\text { fiber directions }\end{array}$ & $\begin{array}{l}\text { Learned from the data; tracking } \\
\text { stops when white/gray matter } \\
\text { boundary is hit }\end{array}$ \\
\hline $\begin{array}{l}\text { FOD with neural } \\
\text { networks }\end{array}$ & \begin{tabular}{|l|} 
Convolutional \\
neural network, \\
high-resolution \\
network, U-Net
\end{tabular} & Not applicable & $\begin{array}{l}\text { Neural networks trained to } \\
\text { regress constrained spherical } \\
\text { deconvolution coefficients }\end{array}$ & Not applicable \\
\hline
\end{tabular}

filtering, image classification and processing, as well as natural language processing. The same approach is also taken in bioinformatics and medical data, helping design new drugs [25], as well as analyze patient scans [26] or genomes [27]. Not surprisingly, it has also been used to track nerve fibers. The use of ML techniques to predict the location of nerve fibers offers numerous benefits. One of the main advantages is the ability to use raw diffusion data directly, without having to represent the diffusion propagator or tissue microstructure [28]. Furthermore, methods of this type are not limited to relying on particular imaging modalities and are capable of learning from different types of DWI experiments.

Additionally, the learning systems may deal with imperfections, such as noise and distortions, and are suitable for identifying the location of white matter tissue in the scan of the entire brain. All that may be learned directly in the model learning phase. The methods discussed are summarized in Table 2, where the algorithms, approaches, key concepts and stopping criteria are presented.

\subsection{Random Forest Classifier}

One of the first approaches relying on ML methodology was proposed by Neher et al. [28]. This method, similarly to traditional approaches, operates in a step-wise fashion when extending the current fiber. However, instead of modeling the signal mathematically, a random forest classifier determines local tissue properties directly from raw diffusion data. During the tracking phase, when moving from a given point onwards, an algorithm considers information from voxels in proximity to a given point. The decision about the new orientation is made in a voting process, with the potential directions sampled from a complete sphere or hemisphere in front of the current position. Classifier output provides the probability for each direction, as well as non-fiber probability. Tracking stops when non-fiber probability exceeds the cumulated weighted probabilities of all possible directions. Otherwise, the streamline direction is calculated as the normalized sum of the proposed directions. The voting process makes the fiber extension process less sensitive to noise and local signal ambiguities, as well as to premature termination.

\subsection{Learn-to-Track}

Neural networks also provide means for analyzing raw diffusion data. Poulin et al. proposed two neural network architectures to accomplish this task [29]. Their feedforward neural network (FFNN) returns a three-dimensional vector describing fiber orientation for each point within the diffusion data. The recurrent neural network (RNN) 
[30] proposed by the authors takes advantage of the previously seen voxels by remembering features relevant to the entire streamline orientation. The authors note that learning a stopping criterion in the neural network approach is not a trivial task, requiring careful engineering and balancing the loss function. Nevertheless, the method was capable of achieving high spatial coverage of a given test set, while simultaneously controlling the number of false positives.

A subsequent study by the group postulates using RNN in a bundle-wise manner, resulting in improved tracking efficiency, a higher number of valid streamlines and better volume coverage in comparison to all classical algorithms [31].

\subsection{DeepTract}

Another method using RNNs was developed by Benou et al. [32] to address fiber orientation estimation as well as to streamline tractography. Like other methods using ML, it is capable of operating well on various types of raw diffusion data. Unlike the previously described methods, it does not produce merely deterministic predictions of the streamline. It estimates the orientation of local fibers as a discrete probability density function, allowing to randomly sample directions at a given point. The authors address the problem of choosing a new direction as a classification task, predicting the probability for each orientation and the probability of tract termination.

\subsection{Neural Network Regression}

A method developed by Wegmayr et al. proposes a multilayer perceptron [33] predicting the outgoing direction from an input. The prediction is made in a cube of a given size, being one of the neural network inputs. The second input is a fixed number of incoming vectors, describing the neural fiber entering the central vector of the cube. The network's output is a vector with three values representing the outgoing direction of the fiber. Tracking is done iteratively and begins with random sampling of the ROI area. Prediction of the initial direction is made with the incoming vector set to zeros. The authors claim that the stopping criteria may be learned from the data itself. However, they have implemented a simple rule to stop tracking when the boundary of white and gray matter is reached.

\subsection{FOD with Convolutional Neural Networks}

Lucena et al. propose to use convolutional neural networks (CNNs), broadly used in image classification, to compute more accurate FOD from a single-shell dMRI [34]. The authors trained two three-dimensional CNNs, a threedimensional high-resolution network (HighResNet) [35] and a three-dimensional U-Net [36] to regress constrained spherical deconvolution coefficients. Although the method does not provide any means of tracking, we decided it was worth mentioning given the produced FODs could be used by other algorithms to track fibers.

\section{Benchmarking Studies}

It is difficult to assess the accuracy of tractography algorithms, especially if predictions are made in vivo, without the possibility to dissect the patient's brain.

In 2015, under an initiative of Klaus H. Maier-Hein, laboratories from across the globe were invited to participate in a tractography competition, where such an assessment was made possible [37]. The methods they relied upon were evaluated based on a specifically crafted data set. It was constructed from multiple whole-brain global tractography maps [38]. A trained radiologist extracted 25 major tracts, comprising association, projection, and commissural fibers. The data prepared for the participants included a structure mimicking clinical-like acquisition of DWI based on a simulated diffusion signal and simulated T1-like contrast. A significant number of methods correctly predicted the topology of most of the fibers under consideration. However, the majority produced, along with correct predictions, many false-positive results, whose number often exceeded that of the correct ones.

A work published by Schilling et al. assesses the accuracy of tractography methods using a data set containing a physical phantom and two ex vivo brain specimens [39]. Although advances in the tractography methods are significant, the authors remark that anatomical accuracy is still limited. This study confirms previous findings showing a great number of false positives generated by the methods. Most algorithms tested had a low connectivity predictive value and low spatial overlap with the true pathways.

\section{Case Study}

Currently, we are designing a neurosurgery support system. It will predict functional cortex regions and white matter fibers in oncological patients. Our goal is to provide predictions based on imaging data to facilitate pre-surgery planning. We would like to propose a new tractography method, being a part of that system, combining a variant of the Dijkstra algorithm [40] with a feedforward neural network backbone. The backbone produces information required to traverse the graph representation of the brain's diffusion map, allowing to trace neural fibers.

The general architecture of the underlying neural network is shown in Fig. 2. In the proposed method, the diffusion data is analyzed in small batches of size $(5,5,5)$. Thus, the required number of nodes in the hidden layers is significantly reduced. A neural network returns a scalar value for each voxel, describing the probability that a given voxel defines the same neural fiber as the central one. Voxels with values above the specified threshold will constitute the nodes of the graph. Weights for edges between the nodes are computed based on the probability values.

Tracking fibers starts by picking the region of interest (ROI), usually covering the area of the planned surgical intervention. Each voxel within ROI is a tracking seed - the 


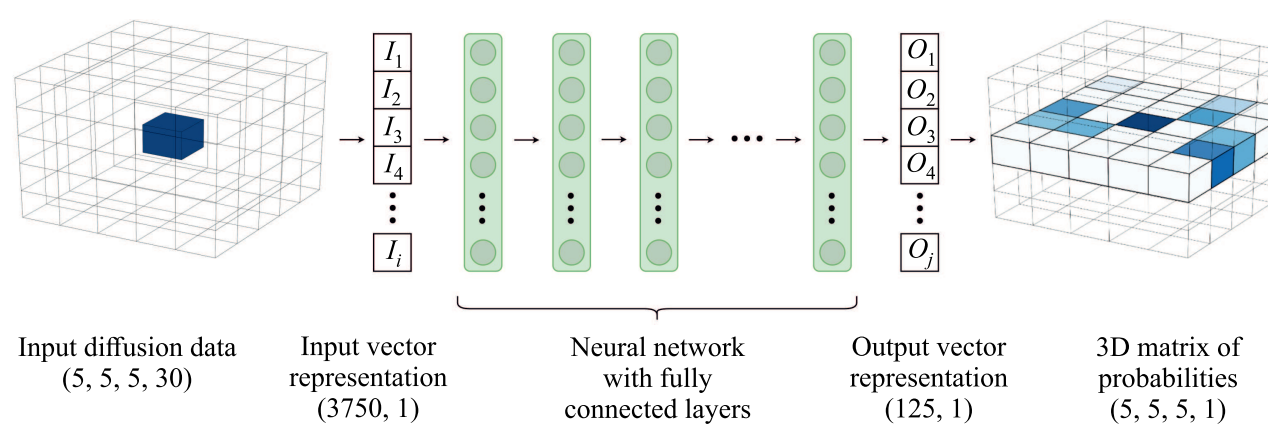

Fig. 2. Architecture of the neural network for predicting probabilities of voxels containing parts of the same fiber.

first node of the graph. In the next step, a three-dimensional matrix of size $(5,5,5)$ with the seed located in the center thereof is drawn. A neural network is employed to produce an output 3D matrix with probabilities for all 125 voxels. The central voxel, as the seeding point, has the probability of 1 (Fig. 2). The graph is extended from the initial node to reach the edge of the drawn cube. The voxel at the border, chosen as the graph node, becomes the central voxel of the next cube. This procedure is presented, on two-dimensional slices, in Fig. 3.

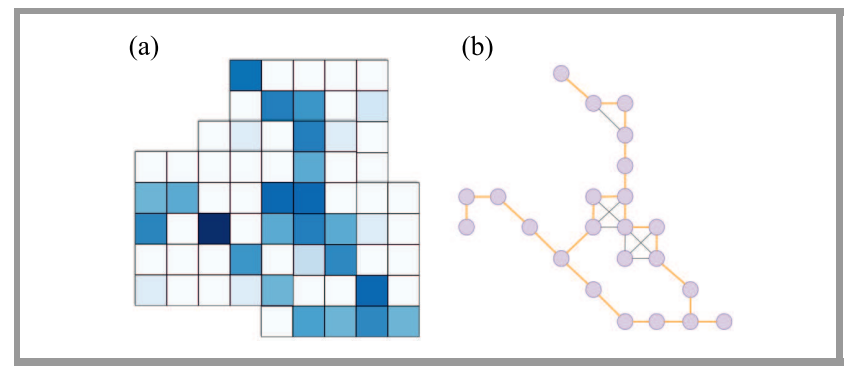

Fig. 3. The method proposed for solving tractography tasks. Part (a) shows path tracking with overlapping cubes obtained from the neural network. Part (b) shows a graph of potential neural paths. Black edges are the possible connections between the nodes. Bold orange edges represent the most probable paths according to the method. (For the color version, please see the digital edition)

In this approach, labeled bands of white matter will quickly cover regions that are remote from the actual ROI. To avoid that, we propose to use two stopping rules. The first is to stop tracking when no satisfying candidates exist near the current node. This principle stems directly from the algorithm used. The additional rule would stop the tracking when a defined Euclidean distance from the seed point is reached.

We are currently collecting data from oncological patients, thanks to long-term cooperation with the Department of Neurosurgery at the Maria Skłodowska-Curie National Research Institute of Oncology. Once this step has been completed, a trained radiology specialist will annotate each diffusion set, producing the expected tractogram. Next, raw diffusion data and tractograms would be used to train the backbone neural network. After the training is completed, the proposed method would be evaluated on the available benchmark dataset. This will allow to compare its accuracy and the rate of false positive predictions with other state-of-the-art methods.

\section{Conclusions}

Diffusion-weighted imaging data, including DTI, is not capable of providing satisfactory answers concerning the location of white matter bands within the brain. To provide such answers, algorithms analyzing the data, developed based on the knowledge of brain anatomy and functions, are used. Throughout the years, many algorithms have been proposed to tackle this problem, including both mathematical and artificial intelligence-based approaches. In this paper, we presented a review of the available methods, with an emphasis placed on their potential use in neurosurgery.

The mathematical models are quite simple in their structure. The final solution of the problem is usually a product of a combination of various methods used together. Each step involves different issues, starting from the noise within the data itself, to complex states on the atomic level that cannot be addressed by such methods alone. These models are developed to process specific diffusion-weighted modalities. The methods based on HARDI imaging data tend to produce smoother, higher resolution tractograms. More information is provided for a single voxel, allowing to adopt a step size that is smaller than the voxel itself. The use of probabilistic models may shed more light on the complex topology of human brain connectomics. However, such methods are prone to produce many false positives, as was shown in the benchmarking studies. The use these methods requires expertise in order to adjust their parameters and to correctly interpret the results at each stage of the analysis. Methods based on ML attempt to bypass at least some of the obstacles encountered by the classical approaches. They may be fed directly with data from imaging experiments to evaluate the solution through model parametrization in the learning process. However, these methods are not completely free of any drawbacks. The process of designing and training a successful model takes a lot of time to complete, thus increasing its total cost. Moreover, a good model 
requires great amounts of well-annotated data. Preparing such a data set is troublesome and labor-intensive. It requires that numerous imaging experiments be conducted with the use of MRI, and that each of them be annotated by a radiology specialist. The effort put into training the model pays off when the method is used. Well-trained models are capable of producing results quickly and without much expertise of the person using them. Therefore, neurosurgeons could use them in an almost out-of-the-box form in preoperative planning.

We propose an approach that is based on a combination of the Dijkstra algorithm with a simple feed-forward neural network be used to predict the most probable fiber topologies within the brain. Such a method would work by performing an iterative analysis of the diffusion data divided into small batches, to speed up the execution process. Continued training and additional experiments are required with real-world patient data in order to optimize the accuracy of the approach and to compare it with other methods using benchmarking datasets.

Despite the tremendous progress made since the introduction of diffusion-weighted imaging, such methods need to be used cautiously and always have to be supported by specific knowledge concerning anatomy and operation of the brain. Novel architectures of neural networks are proposed on a continuous basis to tackle complex tasks in parallel areas, such as natural language processing and image classification. This means that further development of methodologies used to analyze medical data and facilitate diagnostic imaging should be expected as well.

\section{References}

[1] P. A. Young, P. H. Young, and D. L. Tolbert, Basic Clinical Neuroscience. Third Edition. Philadelphia: Lippincott Williams and Wilkins, 2015 (ISBN: 9781451173291).

[2] R. M. Young, A. Jamshidi, G. Davis, and J. H. Sherman, "Current trends in the surgical management and treatment of adult glioblastoma", Annals of Translational Medicine, vol. 3, no. 9, pp. 1-5, 2015 (DOI: 10.3978/j.issn.2305-5839.2015.05.10).

[3] K. D. Merboldt, W. Hanicke, and J. Frahm, "Self-diffusion NMR imaging using stimulated echoes", Journal of Magnetic Resonance (1969), vol. 64, no. 3, pp. 479-486, 1985 (DOI: $10.1016 / 0022-2364(85) 90111-8)$.

[4] D. G. Taylor and M. C. Bushell, "The spatial mapping of translational diffusion coefficients by the NMR imaging technique", Physics in Medicine and Biology, vol. 30, no. 4, pp. 345-349, 1985 (DOI: 10.1088/0031-9155/30/4/009).

[5] J. E. Tanner, "Self diffusion of water in frog muscle", Biophysical Journal, vol. 28, no. 1, pp. 107-116, 1979 (DOI: 10.1016/S0006-3495(79)85162-0).

[6] D. F. Scollan, A. Holmes, R. Winslow, and J. Forder, "Histological validation of myocardial microstructure obtained from diffusion tensor magnetic resonance imaging", American Journal of Physiology - Heart and Circulatory Physiology, vol. 275, no. 6, pp. 2308-2318, 1998 (DOI: 10.1152/ajpheart.1998.275.6.H2308).

[7] M. E. Moseley et al., "Diffusion-weighted MR imaging of anisotropic water diffusion in cat central nervous system", Radiology, vol. 176, no. 2, pp. 439-445, 1990 (DOI: 10.1148/radiology.176.2.2367658).
[8] A. W. Toga et al., "Mapping the human connectome", Neurosurgery, vol. 71, no. 1, pp. 1-€5, 2012 (DOI: 10.1227/NEU.0b013e318258e9ff).

[9] R. G. Briggs et al., "A Connectomic Atlas of the Human CerebrumChapter 18: The Connectional Anatomy of Human Brain Networks", Operative Neurosurgery, vol. 15, no. 1, pp. 470-480, 2018 (DOI: 10.1093/ons/opy272).

[10] S. Mori and P. C. M. Van Zijl, "Fiber tracking: Principles and strategies - A technical review", NMR in Biomedicine, vol. 15, pp. 468480, 2002 (DOI: 10.1002/nbm.781).

[11] B. Jeurissen, M. Descoteaux, S. Mori, and A. Leemans, "Diffusion MRI fiber tractography of the brain", NMR in Biomedicine, vol. 32, no. 4, pp. 1-22, 2019 (DOI: 10.1002/nbm.3785).

[12] P. J. Basser, J. Mattiello, and D. LeBihan, "MR diffusion tensor spectroscopy and imaging", Biophysical Journal, vol. 66, no. 1, pp. 259-267, 1994 (DOI: 10.1016/S0006-3495(94)80775-1).

[13] L. R. Frank, "Anisotropy in high angular resolution diffusionweighted MRI", Magnetic Resonance in Medicine, vol. 45, no. 6, pp. 935-939, 2001 (DOI: 10.1002/mrm.1125).

[14] D. S. Tuch et al. "High angular resolution diffusion imaging reveals intravoxel white matter fiber heterogeneity", Magnetic Resonance in Medicine, vol. 48, no. 4, pp. 577-582, 2002

(DOI: $10.1002 / \mathrm{mrm} .10268)$.

[15] P. J. Basser, "Fiber-tractography via diffusion tensor MRI (DTMRI)", in Proc. of the 6th Annual Meeting ISMRM, vol. 1, 1998, p. 3 [Online]. Available: https://nationalbii.com/wp-content/uploads/ 2020/10/Basser-ISMRM-1998.pdf

[16] M. Lazar et al., "White matter tractography using diffusion tensor deflection", Human Brain Mapping, vol. 18, no. 4, pp. 306-321, 2003 (DOI: 10.1002/hbm.10102).

[17] J. D. Tournier, F. Calamante, and A. Connelly, "MRtrix: Diffusion tractography in crossing fiber regions", Int. J. of Imaging Systems and Technol., vol. 22, no. 1, pp. 53-66, 2012 (DOI: 10.1002/ima.22005).

[18] J. D. Tournier, F. Calamante, and A. Connelly, "Robust determination of the fibre orientation distribution in diffusion MRI: Nonnegativity constrained super-resolved spherical deconvolution", NeuroImage, vol. 35, no. 4, pp. 1459-1472, 2007 (DOI: 10.1016/j.neuroimage.2007.02.016).

[19] M. Descoteaux, R. Deriche, T. R. Knosche, and A. Anwander, "Deterministic and probabilistic tractography based on complex fibre orientation distributions", IEEE Transac. on Medical Imaging, vol. 28, no. 2, pp. 269-286, 2009 (DOI: 10.1109/TMI.2008.2004424).

[20] D. S. Tuch, "Q-ball imaging", Magnetic Resonance in Medicine, vol. 52, no. 6, pp. 1358-1372, 2004 (DOI: 10.1002/mrm.20279).

[21] A. Anwander, M. Tittgemeyer, D. von Cramon, A. Friederici, and T. Knosche, "Connectivity-based parcellation of broca's area", Cerebral Cortex, vol. 17, no. 4, pp. 816-825, 2006 (DOI: 10.1093/cercor/bhk034).

[22] M. A. Koch, D. G. Norris, and M. Hund-Georgiadis, "An investigation of functional and anatomical connectivity using magnetic resonance imaging", NeuroImage, vol. 16, no. 1, pp. 241-250, 2002 (DOI: 10.1006/nimg.2001.1052).

[23] I. Aganj et al., "A Hough transform global probabilistic approach to multiple-subject diffusion MRI tractography", Medical Image Analysis, vol. 15 , no. 4, pp. 414-425, 2011 (DOI: 10.1016/j.media.2011.01.003).

[24] V. J. Wedeen, P. Hagmann, W.-Y. I. Tseng, T. G. Reese, and R. M. Weisskoff, "Mapping complex tissue architecture with diffusion spectrum magnetic resonance imaging", Magnetic Resonance in Medicine, vol. 54, no. 6, pp. 1377-1386, 2005 (DOI: $10.1002 / \mathrm{mrm} .20642$ ).

[25] R. Gupta et al., "Artificial intelligence to deep learning: machine intelligence approach for drug discovery", Molecular Diversity, 2021 (DOI:10.1007/s11030-021-10217-3).

[26] A. Hosny, C. Parmar, J. Quackenbush, L. H. Schwartz, and H. J. W. L. Aerts, "Artificial intelligence in radiology", Nature Reviews Cancer, vol. 18, no. 8, pp. 500-510, 2018 (DOI: 10.1038/s41568-018-0016-5). 
[27] M. W. Libbrecht and W. S. Noble, "Machine learning applications in genetics and genomics", Nature Reviews Genetics, vol. 16, no. 6, pp. 321-332, 2015 (DOI: 10.1038/nrg3920).

[28] P. F. Neher, M. A. Cote, J. Ch. Houde, M. Descoteaux, and K. H. Maier-Hein, "Fiber tractography using machine learning", NeuroImage, vol. 158, pp. 417-429, 2017 (DOI: 10.1016/j.neuroimage.2017.07.028).

[29] P. Poulin et al., "Learn to track: deep learning for tractography", bioRxiv, vol. 1, pp. 540-547, 2017 (DOI: 10.1101/146688).

[30] S. Hochreiter and J. Schmidhuber, "Long short-term memory", Neural Computation, vol. 9, no. 8, pp. 1735-1780, 1997 (DOI: 10.1162/neco.1997.9.8.1735)

[31] P. Poulin, F. Rheault, E. St-Onge, P.-M. Jodoin, and M. Descoteaux, "Bundle-wise deep tracker: Learning to track bundle-specific streamline paths", in Proc. of the Int. Society for Magnetic Resonance in medicine ISMRM-ESMRMB, Paris, France, 2018 [Online]. Available: https://index.mirasmart.com/ISMRM2018/PDFfiles/0041.html

[32] I. Benou and T. R. Raviv, "DeepTract: A Probabilistic Deep Learning Framework for White Matter Fiber Tractography”, D. Shen et al. Eds. in Proc. Medical Image Computing and Computer Assisted Intervention - MICCAI 2019, 22nd Int. Conf., Shenzhen, China, 2019, pp. 626-635 (DOI: 10.1007/978-3-030-32248-9_70).

[33] V. Wegmayr, G. Giuliari, S. Holdener, and J. Buhmann, "Data-driven fiber tractography with neural networks", in Proc. IEEE Int. Symp. on Biomedical Imag. (ISBI), Washington, DC, USA, 2018, pp. 10301033 (DOI: 10.1109/ISBI.2018.8363747).

[34] O. Lucena et al., "Using convolution neural networks to learn enhanced fiber orientation distribution models from commercially available diffusion magnetic resonance imaging", arXiv, 2020 [Online]. Available: https://arxiv.org/pdf/2008.05409.pdf

[35] W. Li, G. Wang, L. Fidon, S. Ourselin, M. J. Cardoso, and T. Vercauteren, "On the compactness, efficiency, and representation of 3D convolutional networks: Brain parcellation as a pretext task. Lecture Notes in Computer Science (including subseries Lecture Notes in Artificial Intelligence and Lecture Notes in Bioinformatics)", in Proc. Int. Conf. on Informat. Process. in Medical Imag., Boone, NC, USA, 2017, pp. 348-360 (DOI: 10.1007/978-3-319-59050-9_28).

[36] O. Cicek, A. Abdulkadir, S. S. Lienkamp, T. Brox, and O. Ronneberger, "3D U-Net: Learning dense volumetric segmentation from sparse annotation”, 2016 [Online]. Available: https://arxiv.org/pdf/1606.06650

[37] K. H. Maier-Hein et al., "The challenge of mapping the human connectome based on diffusion tractography", Nature Communications, vol. 8, no. 1, 2017 (DOI: 10.1038/s41467-017-01285-x).

[38] M. F. Glasser et al., "The human connectome project's neuroimaging approach", Nature Neuroscience, vol. 19, no. 9, pp. 1175-1187, 2016 (DOI: 10.1038/nn.4361).

[39] K. G. Schilling et al., "Limits to anatomical accuracy of diffusion tractography using modern approaches", NeuroImage, vol. 185, pp. 1-11, 2019 (DOI: 10.1016/j.neuroimage.2018.10.029).

[40] E. W. Dijkstra, "A note on two problems in connection with graphs", Numerische mathematik, vol. 1, no. 1, pp. 269-271, 1959 (DOI: 10.1007/BF01386390).

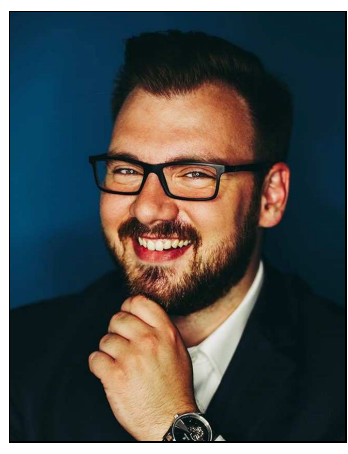

Mateusz Koryciński received his M.Sc. in Bioinformatics from Adam Mickiewicz University Poznań and Poznań University of Technology. $\mathrm{He}$ is currently working at the Department of Bioinformatics and Machine Recognition at the NASK research institute (Warsaw, Poland) and is pursuing a Ph.D. at the Doctoral School of Information and Biomedical Technologies of the Polish Academy of Sciences (Warsaw, Poland). His research interests focus on medical informatics, magnetic resonance imaging and image processing.

(i) https://orcid.org/0000-0002-5239-1635

E-mail: mateusz.korycinski@nask.pl

Department of Bioinformatics and Machine Recognition

Research and Academic Computer Network

ul. Kolska 12

Warsaw, Poland

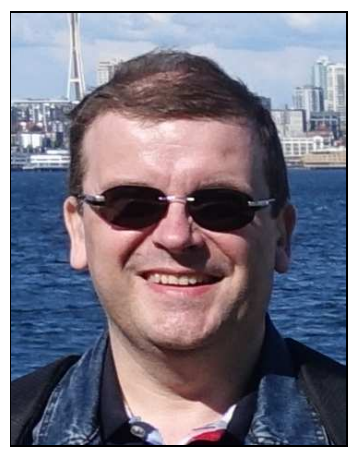

Konrad A. Ciecierski received his Ph.D. (honors) in computer science from Warsaw University of Technology, Poland, in 2014. He specializes in the application of machine learning in medical science, digital signal processing, natural language processing, and deep learning applications. He is currently an assistant professor and head of the Bioinformatics and Machine Recognition department at the NASK research institute. He works at the Clinic of Neurosurgery of the Maria Skłodowska-Curie Memorial Oncology Center in Warsaw, where he is a member of a neurosurgical team specializing in the treatment of the Parkinson disease and other movement-related disorders.

(iD) https://orcid.org/0000-0003-2471-3016

E-mail: konrad.ciecierski@nask.pl

Department of Bioinformatics and Machine Recognition

Research and Academic Computer Network

ul. Kolska 12

Warsaw, Poland 